\title{
Replacement Therapy For Menopause: Effects On Lipid Metabolism
}

\author{
Christina Ruegseggar-Veit, M.D. and Fatma A. Aleem, M.D., Ph. D. \\ New York, New York and Brooklyn, New York
}

DOI: http://dx.doi.org/10.5915/21-4-13516

\begin{abstract}
This review discusses the effect of replacement therapy on lipid metabolism in menopausal women. There is much discussion as to which pharmaceutically-induced lipoprotein changes are of any importance in preventing the development of cardiovascular disease. Hormonal therapy with as few negative lipid metabolism changes as possible is preferable. A parenteral route in which liver passage is avoided may be an advantage.
\end{abstract}

Key wors: Menopause, replacement therapy, lipid metabolism, lipoproteins, cardiovascular disease

For centuries myth and misundestanding have often hampered a scientific approach to the evaluation and treatment of the menopause. Literature dating back to biblical times reflects the evolution of theories about the etiology and sequelae of the menopause. Hippocrates believed that menstrual blood was nourishment for the fetus. Pliny lectured that menstrual blood contained substances to form the offspring. During the past 15 centuries, medical literature has discussed treatment of menopausal women including purgatives, enemas, leeches and phlebotomis. Until the mid-1960s, anecdote and conjecture influenced scientific thought on the climacteric. Not until the emergence of the radioligand assay as a clinically available tool for measuring peptide and steroid hormones has our knowledge on menopause so expanded. This paper discusses primarily the effects of hormone replacement therapy for menopause and its effect on lipid metabolism.

Myocardial infarction (MI) rarely occurs in

From Columbia University College of Physicians and Surgeons, New York, New York, and Brookdale Medical Center, Brooklyn, New York.

Reprint Requests: Fatma A. Aleem, M.D., Ph. D. Department of Clinical Obstetrics and Gynecology Brookdale Hospital Medical Center

1335 Linden Boulevard

Brooklyn, New York 11212 females prior to menopause. Consequently it has been suggested that estrogen might provide a protective effect against atherosclerosis. Younger females who have had bilateral oophorectomy demonstrate a higher incidence of myocardial infarction unless estrogen replacement therapy (ERT) is begun soon after surgery. ${ }^{1}$ However, ERT for prevention of cardiovascular (coronary artery) disease fell into disrepute over a decade ago when men with previous MI were treated with high doses of estrogen in an effort to reduce the risk of recurrent re-infarction. Men given $5 \mathrm{mg}$ daily of conjugated estrogens experienced twice the number of nonfatal MI's, three time the frequency of pulmonary embolus and 1.5 times the number of deaths as did the placebeo group. ${ }^{2}$ However, many studies suggest that estrogens may exert a protective effect against cardiovascular disease. In Lancaster, England, Burch ${ }^{3}$ observed a $63 \%$ decrease in expected deaths from heart disese in 1000 ERT treated women followed for 15 years. Hammond ${ }^{4}$ found a significantly lower incidence of coronary artery disease in ERT treated women as compared to those never using estrogen. Ross et al,' in Lancet 1981, indicated that ERT may protect women against death from ischemic heart disease.

Until a few years ago, post menopausal hormone replacement therapy was based on estrogens alone. There was a general feeling that if estrogen had any effect at all on the risk of coronary heart disease, it was probably beneficial as long as conventional doses of estrogen were used. Many studies have indicated 
that menopause is associated with an increased risk of coronary heart disease. Published data suggested that this risk could be diminished by estrogen substitution. However, it was recommended by Gambrell $^{2}$ that sequential progestogens be added to cyclic estrogen therapy in order to reduce the risk of endometrial cancer. Protection against endometrial hyperstimulation is the main purpose of sequential progesterone treatment in estrogen replacement therapy. Since many of the effects of estrogens, including changes in lipoproteins, are opposed by some progestogens, great interest has been focused on the potential merits and demerits of combining progestogen with estrogen.

\section{Lipoproteins}

Lipoproteins serve as vehicles in the transport of dietary and tissue lipids (cholesterol, triglycerides nd phospolipids in the blood). There are four major classes of lipoproteins in human serum: chylomicrons, VLDL (very low density lipoprotein), LDL (low density lipoprotein), and HDL (high density lipoprotein). Elevated serum LDL cholesterol levels correlate positively with the risk of coronary heart disease; elevated serum VLDL triglycerides also increase this risk. HDL cholesterol is inversely related to coronary heart disease, i.e., it constitutes a protective factor. ${ }^{6}$ Chylomicrons are major carriers of dietary triglycerides from intestine to peripheral tissues. VLDL transports endogenously produced triglycerides to peripheral tissues for consumption and storage. Triglycerides are released by lipoprotein lipase. During this process, VLDL gives rise to small particles that are slowly converted to LDL, which is the principal carrier of cholesterol to the peripheral tissues in man. LDL is removed from the circulation by a receptor medicated process. Both peripheral cells and hepatocytes carry specific membrane receptors that recognize LDL apoprotein.

The formation of HDL, another cholesterol-rich lipoprotein, involves the action of two enzymes: lipoprotein lipase and lecithin cholesterol acyl transferase. HDL particles which contain apoprotein $\mathrm{A}$ are produced in the splanchnic area and are supplied with materials (phospholisids, free cholesterol and apoproteins) derived from the surface of chylomicrons during lipolysis of the trigylcerides by lipoprotein lipase. The HDL particles accomodate the esterified cholesterol in their hydrophobic cores. HDL also receives free cholesterol from peripheral tissues and is involved in reverse transport of cholesterol. Two subfractions of HDL, namely HDL-2 and HDL-3, are found in plasma. The larger HDL-2 particles have four times as much esterified cholesterol as HDL-3, as well as twice the amount of triglycerides and phospholipids.

Hepatic lipase, a lipolytic enzyme with triglyceride lipase and phospholipase activity is located in the en- dothelial cells of liver sinusoids. It participates in catabolism of HDL. Lipid constituents can be released from HDL-2 in a process catalysed by this enzyme with resultant regeneration of HDL-3., ${ }^{7}$

\section{Risk Factors}

One of the most important factors of susceptibility of an individual to atherosclerosis is the concentration of circulating lipids. Cholesterol and triglyceride concentration increase with age. ${ }^{9} \quad$ There is an augmented risk of disease with cholesterol concentrations greater than 220 to $250 \mathrm{mg} / \mathrm{dL} \cdot{ }^{10,12}$ An increase in cholesterol values is associated with increased concentrations of LDL, whereas an increase in triglyceride levels correlates with elevated concentrations of VLDL. HDL, which carries $20 \%$ of cholesterol, seems to protect against the development of atherosclerosis. In the Framingham study, ${ }^{6}$ a low HDL concentration was a more potent risk factor than an elevated concentration of cholesterol or LDL. It is interesting that HDL concentrations are decreased in smoking and increase with regular exercise or alcohol intake. In the Framingham study, there were no deaths from coronary heart disease among 8500 premenopausal females. The odds of death from ischemic heart disease in postmenopausal women in the age range 45 to 54 years were estimated to be 2.4 times those of the premenopausal group. The increased risk of atherosclerotic heart disease after menopause is apparently independent of the risk factors of hyperlipidemia, diabetes mellitus, and hypertension, i.e., these factors were also present in premenopausal women.

\section{Estrogen Replacement Therapy and Risk Factors}

Postmenopausal estrogen replacement therapy was found to be asssociated with beneficial effects on at least two major coronary heart disease risk factors. HDL cholesterol was increased, and LDL cholesterol was decreased by estrogen treatment. ${ }^{9,13-16}$ Tikkanen $^{15}$ demonstrated that the magnitude of the fall in LDL cholesterol was directly proportional to the initial LDL cholesterol level and suggested that estrogen could be the drug of choice in the treatment of postmenopausal hypercholesteremic lipid disorders. Barret-Connor et al " studied 1496 females. After adjusting for the effects of obesity they found that estrogen treated postmenopausal women tended to have lower blood pressure and blood glucose concentrations than did the control subjects. Most investigators have noted an increase in triglyceride levels among estgrogen treated women; Tikkanen ${ }^{15}$ did not observe any significant changes in triglyceride values in females treated for six months with estradiol valerate. Significant protection against atherosclerosis might be provided by an estrogen mediated decrease in cholesterol concentrations and an increase in HDL concentrations. In Tikkamen's 
estrogen treated patients, LDL values were decreased by $18 \%$ and HDL values were increased by $30 \%$. Other data suggest that ERT could actually increase the risk of artherosclerosis in susceptible females through elevation of blood pressure, an increase in LDL concentration, an increased propensity for blood coagulation or an alteration in glucose tolerance. Obviously conflicting data such as these indicated the need for further study.

\section{Estrogen Replacement Therapy and Lipid Metabolism}

There are many problems associated with studying the effects of changes in lipid profiles during and after hormone therapy, especially in that they appear to be subject to numerous variables. These include the type of estrogen, type of progestogen, the dosage of the drug and even the route of administration. A dose of 50 ug of ethinyl estradiol increases both HDL and LDL, while 20 ug of this same estrogen produces an increase in both triglycerides and phospholipids but no change in cholesterol levels. ${ }^{17}$ When a progestogen is added an atherogenic pattern results, i.e., increased LDL, decreased HDL and a marked increase of cholesterol esters. ${ }^{2}$ In contrast, when a socalled "natural" estrogen is administered, e.g., estradiol valerate at $2 \mathrm{mg}$ daily, the triglycerides decrease, and the lipoprotein fractions are altered in reverse to those caused by synthetic estrogens. Both LDL and VLDL decrease while HDL increases, yielding an anti-atherogenic pattern. To add to the confusion, if one increases the dosage of estradiol valerate to $4 \mathrm{mg}$ daily, the pattern of serum lipids becomes similar to that observed with ethinyl estradiol. Conjugated equine estrogens, in dosages less than $2.5 \mathrm{mg}$, decrease serum cholesterol and have no effect on triglycerides. Higher dosages of conjugated estrogens, up to $15 \mathrm{mg}$ a day, have little or no effect on serum cholesterol but increase triglyceride levels. $^{2}$

\section{Progestational Agents and Lipid Metabolism}

Apart from protection against endometrial hyperproliferation, any other effects of progestogen addition to estrogen therapy are undesirable. Therefore it is imperative to know which progestogens to prescribe, i.e., those with a minimum of unwanted side effects. ${ }^{16-22}$ There are two types of progestational agents available: 19-nortestosterone derived progestogens, such as levo-norgestrel, norethindrone and norethindrone acetate, and $\mathrm{C}-21$ progesterone derived from $17-\mathrm{OH}$ progesterone which includes acetates of medroxyprogesterone, megestrol and cyproterone. The former are strongly androgenic, an extermely undesirable side effect. 19-nortestosterone derivatives lower VLDL and triglycerides, whereas 17-OH progesterone do not alter serum triglycerides or VLDL levels when given alone or in combination with estrogen. Although the LDL cholesterol- lowering effects of estrogen replacement is well documented, most progestogens do not cause any significant alterations in LDL cholesterol. However, when norethindorone was given, there was a statistically significant increase in LDL cholesterol after 12 months of therapy. ${ }^{20}$ But not all of the androgenic progestogens have the same effect. The lowering of VLDL lipids by progestogens is obviously beneficial. LDL cholesterol levels are not affected to any major degree. The most consistent lipid effect of postmenopausal progestogen therapy is a reduction in HDL cholesterol by 19-nortestosterone derived progestogens, either alone or in combination with estrogen. Almost all of these progestogens induced an undesired fall in total HDL cholesterol due to a decrease in HDL-2 subfraction. ${ }^{19}$ Relatively nonandrogenic progestogens such as medroxy progesterone acetate and desogestrel do not affect HDL lipid concentrations when used in conventional replacement therapy doses. Ottosson et $\mathrm{al}^{19} \mathrm{com}$ pared synthetic progestogens, 19-nortestostrone and 17-OH progesterone with natural progesterone, i.e., a "micronized" progestone which is effective orally, and their effects on subfractions of HDL cholesterol. Fifty-eight postmenopausal females received three cycles of unopposed ERT; the next three cycles they received levonorgestrel, medroxyl progesterone acetate or natural micronized progesterone during the last ten days of their cycles. Their results supported the evidence that 19-nortestosterone progestogens reversed the effects of estrogen on lipid protein metabolism and decreased the HDL cholesterol concentrations. The 17-OH progesterones did not exhibit such an effect; due to their minimal androgenic effects they were preferred to the former. The natural progesterones had no influence on $\mathrm{HDL}$ cholesterol concentrations.

\section{Effects on Hepatic Metabolism}

Oral estrogens cause marked alternations in hepatic metabolism. These metabolic changes may have important clinical implications as the liver is the major or only source of HDL, LDL, clotting factors and renin substrate, all of which may influence the risk of hypertension, hyprlipidemia, thromboembolic disease and cardiovascular mobidity. Changes in these substances have been related to high hepatic concentration of estrogens after oral administration. Routes of estrogen administration whereby the liver is bypassed, such as vaginal creams and transdermal delivery systems, have been developed. Results of these studies indicate that such delivery routes cause fewer metabolic changes than oral estrogens. ${ }^{23-26}$ Other studies have suggested that the route of administration is not as important as dosage or type of estrogen. De Lignieres et $\mathrm{al}^{23}$ studies 24 postmenopausal women to determine whether the route of administration or type of 
estrogen used is more important in avoiding hepatic function. The women were studied before and at the end of two months of oral or percutaneous administration of the same estrogen: estradiol 17-beta (E-2). The regimens studied were oral micronized E-2 $(2 \mathrm{mg} /$ day $)$ in nine women, oral E-2 valerate $(2 \mathrm{mg} /$ day) in five, and percutaneous E-2 in ten women. Their results indicated that during either oral or percutaneous therapy all patients had relief of their menopausal symptoms within a few days. No significant changes in weight or adverse skin reactions were reported by the group receiving percutaneous E-2. The results were categorized with respect to estrogens, plasma renin substrate, steroid binding protein and lipids. Briefly with regard to estrogens, all three groups demonstrated increased plasma estradiol levels with no significant difference between the groups. A significant decrease in FSH and LH levels was noted in all groups, with no difference between the groups. The plasma renin substrate was increased after oral therapy; no significant change was noted in the percutaneous treatment group. Sex steroid binding protein (sex hormone binding protein, SBP) is a highly sensitive estrogen-inducible serum protein produced by the liver, used to quantify estrogenic potency. This was found to be increased only after oral treatment.

The effects on lipid metabolism were varied. After oral therapy there were no significant changes in the triglyceride level, yet the triglyceride levels were significantly lowered after percutaneous treatment. There were parallel changes in the VLDL levels in all groups. No demonstrable alterations in the levels of total cholesterol, HDL and LDL were documented after treatment by any of the three groups. It was concluded that both oral and percutaneous E-2 administration are efficient methods of ERT (i.e., increased estradiol levels and lowered $\mathrm{FSH}$ and $\mathrm{LH}$ concentrations). There was an improvement in postmenpausal symptoms with all regimens. There appears to be an enhanced sensitivity to oral estrogen therapy. Perhaps this is due to the resultant higher intrahepatic concentration of estrogen after oral therapy; the parenteral route may result in greater peripheral dilution and relatively lower hepatic concentrations. Sex hormone binding protein did not change after E-2 percutaneous therapy. Fahraeus ${ }^{26}$ reported similar results. It may be, as postulated by De Lignieres, that the SBP is a marker which reflects the hepatic action of oral estrogens. The increase in plasma renin substrate is an adverse side effect from clinical standpoint, as it can lead to hypertension. Here, plasma renin substrate was increased after oral therapy. No changes occured after percutaneous therapy. A slight increase in HDL concentrations was noted in all groups, however it was not statistically significant. Fahraeus, on the other hand, reported a significant increase in HDL during oral E-2 therapy alone. This metabolic change was considered beneficial because of the generally acceptable inverse correlation between HDL levels and cardiovascular risk. One of the conclusions of De Lignieres' study was that percutaneous E-2 therapy relieves climacteric symtoms without consistently altering liver protein production. Ottsson ${ }^{17}$ studied the effects of natural and synthetic hormones on the subfractions of HDL and liver proteins. Postmenopausal women were followed during replacement therapy with various estrogen/progestogen combinations. Three cycles of unopposed estrogen therapy were followed by three cycles of estrogen therapy and 10 days of levonorgestrel, medroxyl progeserone acetate or natural micronized progesterone. The total serum cholesterol was significantly reduced in all regimens. Apolipoprotein A, a major component of HDL, has been suggested to be a more important indicator than HDL of the risk of ischemic heart disease. In the Ottosson study, the concentrations of HDL, HDL-2, apoprotein A-1 and A-2 were all elevated in a dose dependent pattern during unopposed estrogen therapy. HDL and HDL-2 were decreased in the cycles treated with the synthetic progesterones. However, treatment with natural progesterone revealed no changes in HDL or its subfractions.

\section{Summary}

There is much discussion as to what pharmaceutically-induced lipoprotein changes are of any importance in the prevention of the development of cardio-vascular disease. Recent reports of beneficial effects of lipid correction in long term estrogen/progesterone replacement therapy would indicate this direction. The lipid responses also depend on the route of administration. Oral therapy with micronized ertradiol decreases LDL and increases HDL due to raised HDL-2 levels, while percutaneous therapy with estradiol produces only minor lipid changes. A lesser decrease in HDL has been observed when conjugated estrogens were combined with $\mathrm{C}-21$ progestogens such as medroxy progesterone acetate. Whitehead ${ }^{27}$ recommends a regimen of daily conjugated estrogens (Premarin $0.625 \mathrm{mg}$ o.d.) followed by 12 days of $5 \mathrm{mg}$ medroxy progesterone acetate or $0.35 \mathrm{mg}$ norethindrone. It has been suggested that, based on all the available data, the use of natural oral estrogens combined with C-21 progesterones is the preferred treatment for postmenopausal replacement therapy. A hormonal therapy with as few negative lipid metabolism changes as possible is preferable. A parenteral route in which first liver passage is avoided may be an advantage. The beneficial effects of transdermal estrogen therapy are evident and further study may prove this to be the most effective means of replacement therapy. 


\section{References}

1. Notelovitz M, Gudat JC, Ware MD, Dougherty MC: Lipids and lipoproteins in women after oophorectomy and the response therepy. $\mathrm{Br} \mathrm{J}$ Obstet Gynecol 1983;90(2):171-177.

2. Gambrell RD: The menopause: Benefits and risks of estrogen-progestogen replacement therapy. Fertil Steril 1982;37:457-474.

3. Burch JC, Byrd BV, Vaughn WK: Results of estrogen treatment in 1000 hysterectomized women for 14,318 years. In: Consensus on Menopausal Research. Edited by PA van Keep. RB Greenblatt, M Albeaux-Fernet. Lancaster, England: MTP Press 1976, p.164.

4. Hammond CB, Jelovsek FR, Lee KL, Creasman WT, Parker RT: Effects of long term estrogen replacement therapy: Metabolic effects. Am J Obstet Gynecol 1979;133:525.

5. Ross RK, Paganini-Hill A, Mack TM, Arthur M, Henderson BE: Menopausal estrogen therapy and protection from death from ischemic heart disease. Lancet;1981(1):858.

6. Gordon T, Castelli WP, Hjortland MC, Kannel WB, Dawber TR: High density lipoprotein as a protective factor against coronary heart disease: The Framingham Study. Am J Med 1977;62: 707-714.

7. Utian WH, Gordan GS: Metabolic changes due to menopause and their response to estrogen. In: Female and Male Climacteric, edited by PA van Keep, DM Serr, RB Greenblatt. Lancaster, England: MTP Press 1979, P.195.

8. Cauley JA, LaPorte RE, Kuller LH, Bates M, Sandler RB: Menopausal estrogen use high density lipoprotein cholesterol subfractions and liver function. Atherosclerosis 1983;49(1):31-39.

9. Crona N, Silfverstolpe G, Samsioe G: The influence of hormonal replacement therapy on lipid and lipoprotein metabolism. Acta Obstet Gynecol Scand 1985;9(suppl 130):49-52.

10. Cauley JA, LaPorte RE, Kuller LH, BlackSandler R: The epidemiology of high density lipoprotein cholesterol levels in post-menopausal women. J Gerontol 1982;34 (1):10-15.

11. Barrett-Connor E, Brown V, Turner J, Austin $\mathrm{M}$, Criqui $\mathrm{MH}$ : Heart disease risk factors and hormone use in postmenopausal women. JAMA 1978;241:2167.

12. Stampfer MJ, Willett WC, Colditz GA, Rosner B, Speizer FE, Hennekens $\mathrm{CH}$ : A prospective study of postmenopausal estrogen therapy and coronary heart disease. $\mathrm{N}$ Eng1 J Med 1985;313(17):1044-1049.

13. Hart DM, Farish E, Fletcher CD, Howie C, Kitchener $\mathrm{H}$ : Ten years post-menopausal hormone replacement therapy-Effect on lipoproteins. Maturitas 1984;(4):271-276.

14. Barnes RB, Roy S, Lobo RA: Comparison of lipid and androgen levels after conjugated estrogen or depo-medroxyprogesterone acetate treatment in post-menopausal women. Obstet Gynecol 1985;66(2):226-219.

15. Tikkanen MJ, Nikkita EA, Vartiainen E: Natural Estrogen as an effective treatment for Type II hyperlipo-proteinemia in postmenopausal women. Lancet; 1978(2):490.

16. Schaefer EJ, Foster DM, Zech LA, Lindgren FT, Brewer HB, Levy RI: The effects of estrogen administration on plasma lipoprotein metabolism in premenopausal females. J Clin Endo Metab $1983 ; 57: 262-267$.

17. Ottosson UB: Oral progesterone and estrogen/progestogen therapy. Effects of natural and synthetic hormones on subfractions of HDL cholesterol and liver proteins. Acta Obstet Gynecol Scand 1984;9(s 127):1-37.

18. Christiansen C, Christiansen MS, Grande P, Transbol I: Low-risk lipoprotein pattern in postmenopausal women on sequential estrogen/ progestogen treatment. Maturitas 1984;5(3): 193-199.

19. Ottosson UB, Johnson BG, von Schoultz: Subfractions of high density lipoprotein cholesterol during estrogen replacement therapy: A comparison between progestogens and natural progesterone. Am J Obstet Gynecol 1985;151(6): 746-750.

20. Yikkanen MJ, Kussi T, Nikkila EA, Sipinen S: Post-menopausal hormone replacement therapy: Effects of progestogens on serum lipids and lipoproteins. A review. Maturitas 1986;8(1):7-17.

21. Hirvonen MJ, Malkonen M, Manninen V: Effects of different progestogens on lipoproteins during post-menopausal replacement therapy. N Eng1 J Med 1981; 304(10):560-563..

22. Ottosson UB, Carlstrom K, Johansson BG, von Schoultz B: The effects of an antiestrogen on subfractions of HDL cholesterol during estrogen replacement therapy. Gynecol Obstet Invest 1984; 18(3):140-146.

23. De Lignieres B, Basdevant A, Thomas G, Thalabard JC,Mercier-Bodard C, Conrad J, Guyene TT, Marion N, Corvol P, Guy-Grand B: Biological Effects of estradiol-17 beta in postmenopausal women: Oral versus percutaneous administration. J Clin Endocrinol Metab 1986;62(3):536-541.

24. Basdevant A, De-Lignieres B, Guy-Grand B: Differential lipemic and hormonal response to oral and parenteral 17 beta-estradiol in postmenopausal women. Am J Obstet Gynecol 1983;147(1):77-81.

25. Sharf M, Oettinger M, Lanir A, Kahana L, Veshrun D: Lipid and Lipoprotein levels following pure estradiol implantation in postmenopausal women. Gynecol Obstet Invest 
1985;19(4):207-211.

26. Fahraeus L, Wallentin L: High density lipoprotein subfractions during oral and cutaneous administration of 17 beta-estradiol to menopausal women. J Clin Endo Metab 1983;56(4):797-801.
27. Padwick ML, Pryse-Davies J, Whitehead M: A simple method for determining the optimal dosage of progestins in postmenopausal women receiving estrogens. N Eng J Med 1987;315(15): 930. 